\title{
A Unified Analysis of PWM Converters in Discontinuous Modes
}

\author{
Dragan Maksimović, Member, IEEE, and Slobodan Ćuk, Member, IEEE
}

\begin{abstract}
Three discontinuous operating modes of PWM converters are considered in this paper-the discontinuous inductor current mode (DICM), the discontinuous capacitor voltage mode (DCVM) and a previously unidentified mode named discontinuous quasi-resonant mode (DQRM). DC and smallsignal ac analyses are applicable to all basic PWM converter topologies. Any particular topology is taken into account via its well-known dc conversion ratio in the continuous conduction mode. The small-signal model is of the same order as the statespace averaged model for the continuous mode, and it offers improved predictions of the low-frequency dynamics of PWM converters in the discontinuous modes. It is shown that converters in discontinuous modes exhibit lossless damping similar to the effect of the current-mode programming.
\end{abstract}

\section{INTRODUCTION}

$\mathrm{B}_{\mathrm{a}}^{\mathrm{a}}$ ASIC PWM (square-wave) converter topologies, such as buck, boost, buck-boost, Ćuk, and their versions with an isolation transformer, are most widespread in dcto-dc conversion applications. The most common operating mode of PWM converters is the continuous conduction mode (CCM), in which two networks are repeatedly switched by the action of the power-stage switches. It is also well known that CCM is not the only possible operating mode. Three networks are periodically switched in the discontinuous inductor current mode (DICM) and in its dual-the discontinuous capacitor voltage mode (DCVM) [1]

Steady-state and dynamic properties of the PWM power stage can be vastly different depending on the operating mode. For CCM, the method of state-space averaging [2] yields a general dc model and a general small-signal ac model that have been successfully utilized in practice. In [3], the method was extended to DICM, while in [1], the results for DCVM were deduced by duality.

The purpose of this paper is to provide a systematic classification of possible operating modes of PWM converters, and to complete the understanding of dc conver-

\footnotetext{
Manuscript received September 20, 1989. This paper was presented at the 1989 International Power Conversion and Intelligent Motion Conference, Munich, West Germany. June 6-9. This work was conducted under the Power Electronics Program supported by grants from Boeing Electronics Company, GTE Communication Systems Corp., Rockwell, Inc., and $\mathrm{EG} \& \mathrm{G}$ Almond Instruments, Inc.

D. Maksimovic is with the Faculty of Electrical Engineering, University of Belgrade, Belgrade, Yugoslavia.

S. Cuk is with the Power Electronics Group, California Institute of Technology, Pasadena, CA 91125

IEEE Log Number 9100527.
}

sion properties and low-frequency dynamics of PWM converters in discontinuous modes.

In Section II, it is shown that in addition to CCM, DCIM and DCVM, yet another discontinuous mode is possible. The discontinuous quasi-resonant mode (DQRM) can be viewed as a combination of the two known discontinuous modes. A general dc model is described in Section III and applied to each of the three discontinuous modes. For a given PWM converter, unified expressions yield the dc conversion ratio and the boundary condition between the continuous and the discontinuous mode. An averaged small-signal ac model is derived in Section IV together with closed-form expressions for the model's parameters. Implications of the ac model are discussed with respect to the earlier, reduced-order statespace averaged model [3], and with respect to some general limitations of averaged ac models. The ac model is verified by frequency response measurements of experimental PWM converters operating in the discontinuous inductor current mode. Properties of PWM converters in the discontinuous modes are summarized in Section V.

\section{Operating Modes of PWM Converters}

In this paper, we consider a class of basic PWM converters with two switches (a single transistor and a single diode), and an arbitrary number of reactive elements (capacitors and inductors). It is assumed that all elements in the converter are ideal. The basic converters include all well-known topologies such as buck, boost, buck-boost, Cuk, Sepic, etc., and the results can be easily extended to the topologies such as flyback, forward, Cuk with an isolation transformer etc., derived from the basic converters by insertion of an isolation transformer.

Consider, as an example, the buck converter with input filter in Fig. 1. In the continuous conduction mode (CCM), the diode switching is synchronous with the transistor switching-when the transistor is oN, the diode is OFF and vice versa. Thus, two linear, time-invariant networks (operating states) are repeatedly switched in CCM. If the operating states are denoted according to the position of the transistor and the diode switch (ON $\equiv 1$, OFF $\equiv 0$ ), CCM is described by the following periodic sequence of states:

$$
\cdots \rightarrow 1-0 \rightarrow 0-1 \rightarrow \cdots .
$$




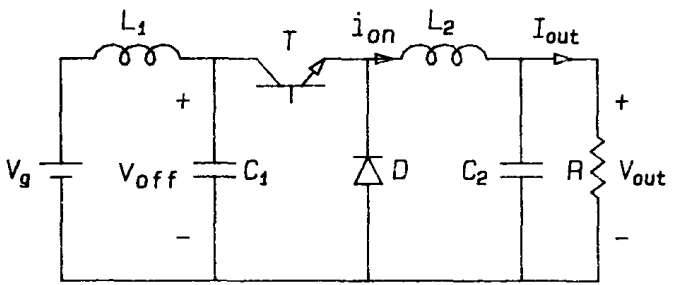

Fig. 1. Buck converter with input filter

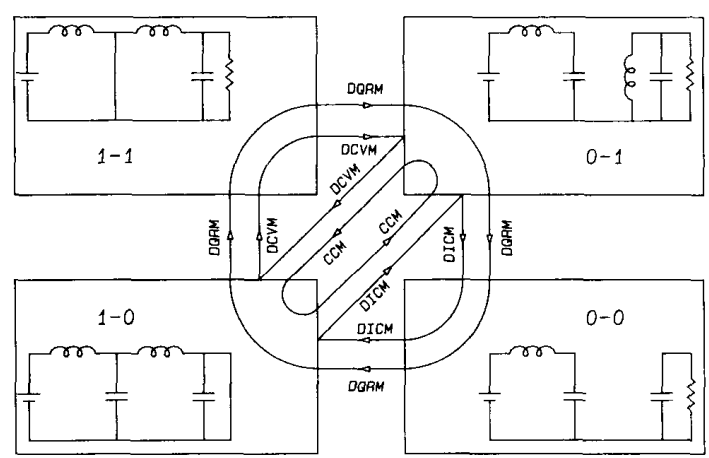

Fig. 2. Loops in diagram of operating states represent possible operating modes.

The periodic sequence of states in CCM defines a loop in the diagram of Fig. 2 where all four possible states are shown.

For the operation in CCM, it is necessary that the diode current is positive during the transistor ofF-time and that the diode voltage is positive during the transistor on-time. The operation in CCM is guaranteed under the small-ripple assumption that all steady-state inductor currents and capacitor voltages are dc quantities with a relatively small superimposed ac ripple. In Fig. 3(a), voltage $v_{\text {off }}$ across the input filter capacitor $C_{1}$ and current $i_{\text {on }}$ through the output filter inductor $L_{2}$ are shown with the small ac components represented by approximate, piecewise linear waveforms.

Let us now examine what could happen if the smallripple assumption is selectively removed from the energystorage elements in the buck converter with input filter. Removing the small-ripple assumption from a capacitor (or an inductor) can be regarded as reducing the value of its capacitance (inductance), while all other parameters (input voltage, load, switching frequency, duty ratio) remain the same.

The values of the $C_{2}$ capacitance and the $L_{1}$ inductance do not affect the operating mode of the converter. Even if the output capacitor $\left(C_{2}\right)$ is completely removed from the circuit, or if the input inductor $\left(L_{1}\right)$ is shorted, the switch voltage/current waveforms are unaltered, so that the converter remains in the continuous conduction mode.

Suppose that the small-ripple assumption is removed from the output inductor $L_{2}$. If the ac ripple of the output inductor current is sufficiently large, the diode will cease to conduct before the end of the transistor OFF-time, as illustrated in the waveforms of Fig. 3(b). In the resulting operating mode, which is well-known as the discontinuous inductor current mode (DICM), three operating states are periodically switched:

$$
\cdots \rightarrow 0-0 \rightarrow 0-1 \rightarrow 0-0 .
$$

The loop corresponding to the sequence of operating states in DICM is shown in Fig. 2.

If the small-ripple assumption is removed from the filtering capacitor $C_{1}$, the ac ripple in its voltage can be sufficiently large so that the diode starts to conduct before the end of the transistor on-time. The resulting mode is called the discontinuous capacitor voltage mode (DCVM). In [1], where DCVM was first uncovered, it was shown that DCVM and DICM are dual operating modes. The waveforms typical for DCVM are shown in Fig. 3(c), and the three periodically switched operating states,

$$
\cdots \rightarrow 1-0 \rightarrow 1-1 \rightarrow 0-1 \rightarrow \cdots,
$$

yield another loop in the diagram of Fig. 2.

The remaining possibility is to remove the small-ripple assumption from both $L_{2}$ and $C_{1}$. This option, which quite naturally follows from the preceding discussion, yields a previously neglected operating mode of PWM converters. The mode will be called the discontinuous quasi-resonant mode (DQRM) because $L_{2}$ and $C_{1}$ form a resonant circuit in one of the operating states. The sequence of operating states in DQRM,

$$
\cdots \rightarrow 1-0 \rightarrow 1-1 \rightarrow 0-1 \rightarrow 0-0 \rightarrow \cdots,
$$

is illustrated in Fig. 2, where it is apparent that DQRM can be considered as a combination of DICM and DCVM. Typical waveforms shown in Fig. 3(d) can be used as a reference for a qualitative description of the operation in DQRM. Assume that initially the converter is in the (0-0)-state-both the transistor and the diode are ofF. At the beginning of a switching cycle, the transistor is turned ON and the resonant (1-0)-state is entered. Note that the transistor current is equal to zero before and immediately after the turn-on transition or, as we shall say from now on, the transistor is turned oN at zero current. In the (1-0) state, voltage $v_{\text {off }}$ and current $i_{\text {on }}$ evolve in a quasi-sinusoidal manner until $v_{\text {off }}$ reaches zero and the diode starts to conduct at zero voltage. The converter remains in the (1-1)-state until the transistor is turned ofF at zero voltage, and the (0-1)-state is entered. In the (0-1)-state, the diode current decays linearly, while the transistor voltage ramps up linearly. The remaining (0-0)-state is entered when the diode current drops to zero. The converter stays in the (0-0)-state until the transistor is turned-on again and the switching cycle is completed.

In DQRM, the transistor is turned on at zero current 


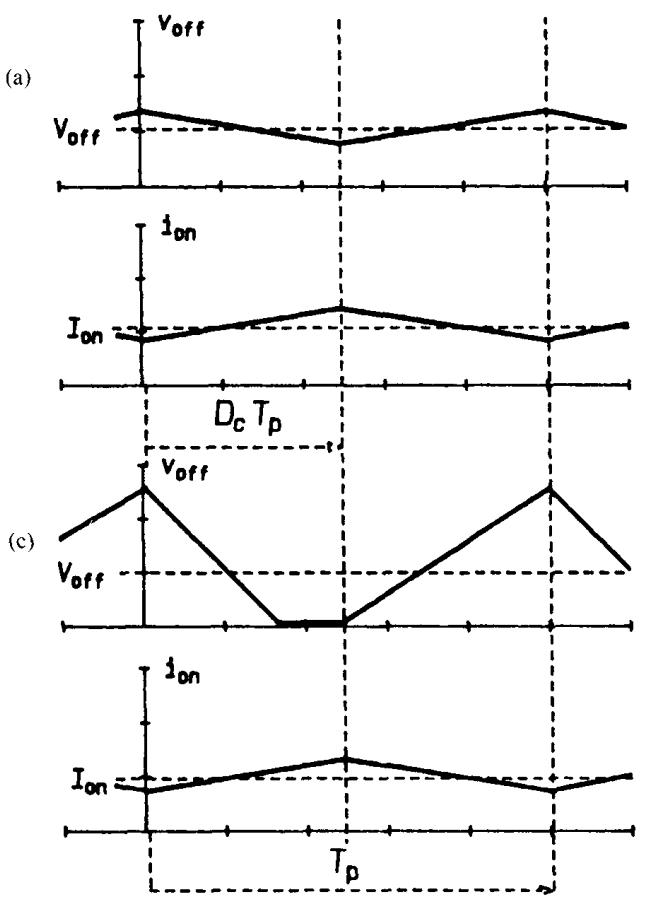

Fig. 3. Waveforms of $v_{\text {off }}$ and $i_{\mathrm{cm}}$ in (a) CCM; (b) DICM; (c) DCVM; and (d) DQRM

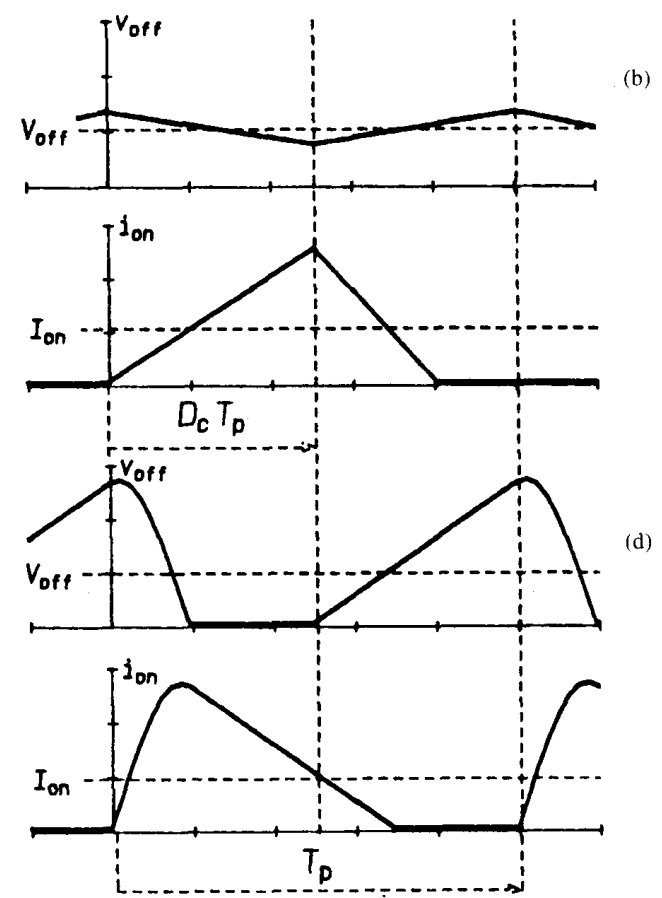

and OFF at zero voltage. On the other hand, the diode is turned on at zero voltage and OFF at zero current. Thus, in a PWM converter operating in DQRM, switching losses associated with semiconductor devices are reduced in the same manner as in various quasi-resonant converters [5], [6]. In fact, in [7] it is shown that PWM converters operating in DQRM can also be considered as members of additional quasi-resonant classes. As opposed to other quasi-resonant converters, which require a variable-frequency control, PWM converters in DQRM can be controlled by varying the transistor duty ratio at constant switching frequency, just as PWM converters in CCM. Also, the active switch in DQRM need not be bidirectional.

So far, in the discussion of possible operating modes of PWM converters, we referred to one particular examplethe buck converter with input filter. Now, the study of possible operating modes can be put into a quite general framework. In [8], it was shown that the transistor and the diode in every PWM converter form:

- a loop with a (possibly empty) set of capacitors and possibly $V_{g}$;

- a cut-set with a non-empty set of inductors.

The set of the capacitors in the loop with the switches is denoted by $\mathcal{C}_{\text {off }}$, while the set of inductors in the cut-set with the switches is denoted by $\mathscr{L}_{\text {on }}$. Voltage $v_{\text {off }}$ is the sum of voltages across the capacitors in $\mathfrak{C}_{\text {off }}$, including the input voltage source, if $V_{g}$ is in the loop. The reference orientation is chosen so that $v_{\mathrm{off}}$ is non-negative. In the example of Fig. 1, there is only one capacitor $\left(C_{1}\right)$ in $\mathrm{C}_{\text {off }}$, so that $v_{\text {off }}=v_{C l}$. Current $i_{\text {on }}$ is the sum of the currents through the inductors in $\mathfrak{L}_{\text {on }}$ with the reference orientation chosen so that $i_{\text {on }}$ is non-negative. In the buck converter with input filter, only $L_{2}$ is in $\mathscr{L}_{\text {on }}$, so that $i_{\text {on }}=$ $i_{L 2}$.

In order to identify the capacitors in $\mathcal{C}_{\text {off }}$ (and possibly $V_{g}$ ), one need only find all the elements that contribute to the switch voltage stress when the switch is OFF. Similarly, currents of all inductors in $\mathscr{L}_{\text {on }}$ contribute to the switch current stress when the switch is oN.

After variables $v_{\text {off }}$ and $i_{\text {on }}$ are defined, possible operating modes of PWM converters can be characterized as follows:

- In CCM, voltage $v_{\text {off }}$ and current $i_{\text {on }}$ are positive for all $t$. A sufficient condition for operation in CCM is that the small-ripple assumption holds true for all capacitors and inductors in the converter.

- In DICM, current $i_{\text {on }}$ is zero during a part of the switching cycle, while $v_{\text {off }}>0$ for all $t$. The smallripple assumption is invalid for at least one inductor in $\mathfrak{L}_{\text {on }}$.

- In DCVM, voltage $v_{\text {off }}$ is clamped to zero during a part of the switching cycle, while $i_{\text {on }}<0$ for all $t$. The small-ripple assumption is invalid for at least one capacitor in $\mathcal{C}_{\text {off }}$.

- In DQRM, there is an interval when $v_{\text {off }}=0$ and an interval when $i_{\text {on }}=0$ in every switching cycle. The small-ripple asumption is invalid for at least one capacitor in $\mathcal{C}_{\mathrm{off}}$ and for at least one inductor in $\mathcal{L}_{\mathrm{on}}$. 


\section{DC Analysis}

In this section, steady-state properties of the three discontinuous modes of PWM converters are examined. A general analysis approach is outlined in Section IV-A and then applied to DICM, DCVM and DQRM in Sections IV-B through IV-D.

\section{A. A Unifying Analysis Method}

In CCM, the dc conversion ratio $\mathfrak{M}$ is a function of the duty ratio $D$ of the transistor switch,

$$
\mathfrak{T} \equiv \frac{V_{\text {out }}}{V_{g}}=M(D)=\frac{P(D)}{Q(D)}
$$

where $P(D)$ and $Q(D)$ are polynomials in $D$. For example, $M(D)=D$ for the buck converter, $1 /(1-D)$ for the boost converter, and $D /(1-D)$ for the buck-boost, Cuk and Sepic converters.

Define the equivalent duty ratio $m$ as the ratio of the average diode voltage and the average of voltage $v_{\text {off }}$,

$$
m \equiv \frac{\bar{v}_{d}}{\bar{v}_{\text {off }}} \equiv \frac{\bar{v}_{d}}{V_{\text {off }}}
$$

It is easy to see that $m=D$ in CCM. For any other operating mode (DICM, DCVM, DQRM), the equivalent duty ratio can be found as a function of circuit parameters, control variables, and the ratio $V_{\text {off }} / I_{\mathrm{on}}$,

$$
m=m\left(f_{s}, D_{c}, \frac{V_{\mathrm{off}}}{I_{\mathrm{on}}}\right) .
$$

In order to make a distinction, the transistor duty ratio is denoted by $D_{c}$ in discontinuous modes, whereas symbol $D$ is reserved for the transistor duty ratio in the continuous conduction mode. In general, either the switching frequency $\left(f_{s}\right)$ or the transistor duty ratio $\left(D_{c}\right)$ or both could be used as control variables in the discontinuous modes. We shall restrict our attention to the case when $D_{c}$ is the control variable, while $f_{s}$ is constant and treated as a circuit parameter.

If $m$ is known, the conversion ratio $\mathfrak{T}$ can be found simply by replacing the duty ratio $D$ with the equivalent duty ratio $m$ in the well-known function $M(D)$ for the PWM converter CCM:

$$
\mathfrak{M}=M(m) .
$$

The result (8) is not immediately useful because the equivalent duty ratio is a function of the converter's unknown internal variables $V_{\text {off }}$ and $I_{\text {on }}$. However, for every PWM converter under consideration, it can be shown that [9]:

$$
\begin{gathered}
\frac{V_{\text {off }}}{V_{g}}=\frac{1}{Q(D)}, \\
\frac{I_{\text {on }}}{I_{\text {out }}}=\frac{1}{Q(D)} .
\end{gathered}
$$

TABLE 1

EQUivalent DUty Ratio $m$ AS A FUNCTION OF THE CONVERSION RATHO $M$ FOR THE THREe MOST COMMON Conversion-Ratio Functions $M(D)$ IN $\mathrm{CCM}$

\begin{tabular}{cc}
\hline$M(D)$ & $m(\mathfrak{K})$ \\
\hline$D$ & $\mathfrak{K}$ \\
$\frac{1}{1-D}$ & $\frac{\mathfrak{M}-1}{\mathfrak{K}}$ \\
$\frac{D}{1-D}$ & $\frac{\mathfrak{K}}{\mathfrak{K}+1}$ \\
\hline
\end{tabular}

Then, it follows that

$$
\frac{V_{\text {off }}}{I_{\text {on }}}=\frac{V_{g}}{I_{\text {out }}},
$$

so that the equivalent duty ratio becomes a function of external variables only,

$$
m=m\left(D_{c}, \frac{V_{g}}{I_{\text {out }}}\right)
$$

For a given operating mode, $m$ is the same for any PWM topology. If $M(D)$ in CCM is known, the conversion ratio in a discontinuous mode can be determined from (8). Conversely, if the conversion ratio is known, the equivalent duty ratio can be found by inverting (8). In Table I, the equivalent duty ratio is found as a function of $\mathfrak{M}$ for the three most common functions $M(D)$. A general dc model of PWM converters is shown in Fig. 4. The duty ratio $D$ is replaced by the equivalent duty ratio, and the $\mathrm{dc}$ model in CCM is augmented by the block where the equivalent duty ratio is computed. The function implemented in the block depends on the operating mode but not on the PWM topology. The general model of Fig. 4 implies that PWM converters that share the same conversion ratio in CCM,

$$
\mathfrak{K}=M(D),
$$

will share the same conversion ratio

$$
\mathfrak{K}=M(m)=M\left(D_{c}, V_{g}, I_{\text {out }}\right)
$$

in a discontinuous mode.

With a somewhat different interpretation, the unifying analysis method outlined in this section was first proposed in [8] where unified analyses of two classes of quasi-resonant converters were completed.

\section{B. Discontinuous Inductor Current Mode}

The waveform of current $i_{\text {on }}$ in DICM is shown in Fig. 5 together with a notation for all relevant time intervals. During the period $D_{c} T_{p}$ when the transistor switch is oN, voltage across every inductor in $\mathscr{L}_{\text {on }}$ is equal to $V_{\text {off }}-\bar{v}_{d}$. When the transistor switch is ofF, voltage across every inductor in $\mathfrak{L}_{\text {on }}$ is equal to $-\bar{v}_{d}$, as long as the diode current is positive. Assuming that the diode ceases to con- 


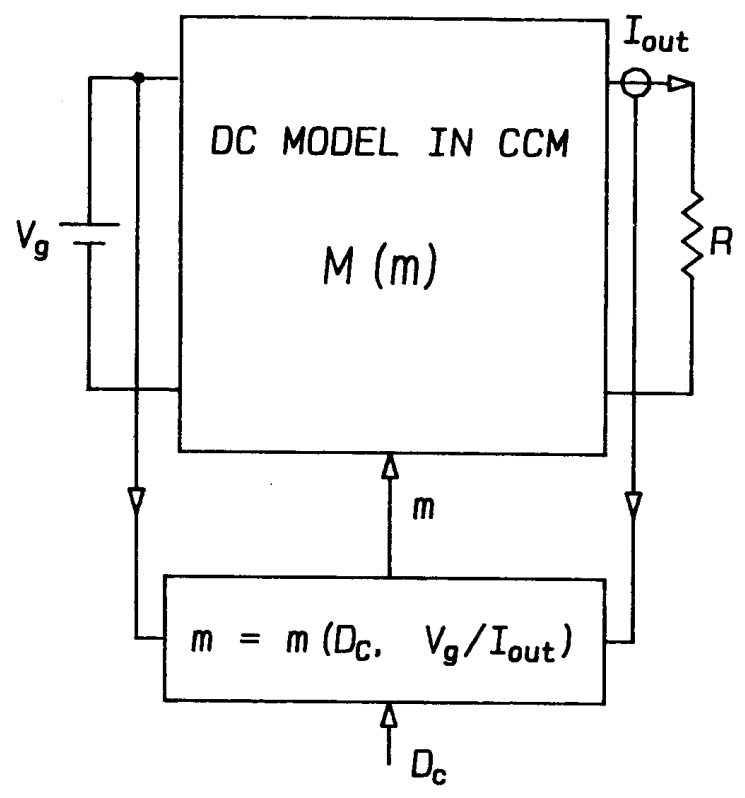

Fig. 4. General dc model of PWM converters.

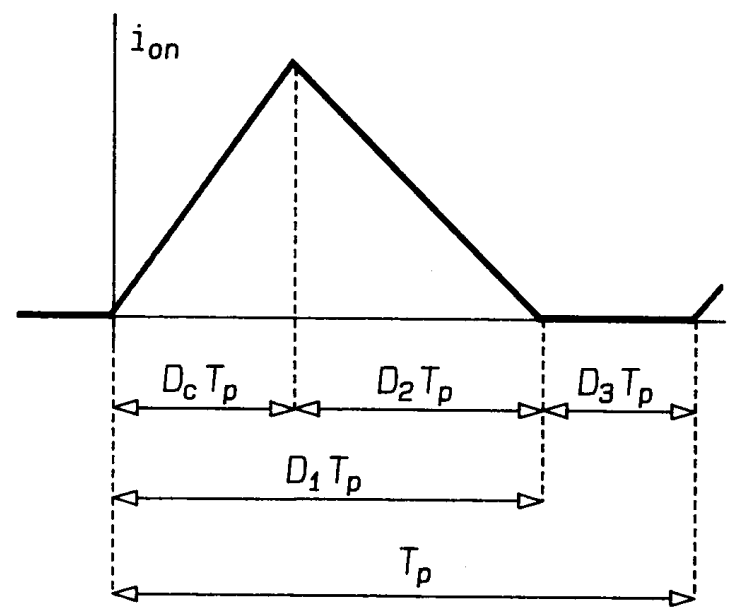

Fig. 5. Typical waveform of current $i_{\mathrm{on}}$ in discontinuous inductor current mode.

duct before the end of the switching cycle, we have

$$
\begin{aligned}
L_{e} \frac{d i_{\mathrm{on}}}{d t} & =V_{\mathrm{off}}-\bar{v}_{d}, \quad 0<t \leq D_{c} T_{p}, \\
L_{d} \frac{d i_{\mathrm{on}}}{d t} & =-\bar{v}_{d}, \quad D T_{p}<t \leq D_{1} T_{p}, \\
i_{\text {on }} & =0, \quad D_{1} T_{p}<t<T_{p} .
\end{aligned}
$$

Equivalent inductance $L_{e}$ is the inductance of the parallel combination of inductors in $\mathscr{L}_{\text {on }}$,

$$
\frac{1}{L_{e}} \equiv \sum_{\mathcal{L}_{\mathrm{on}}} \frac{1}{L_{i}}
$$

Now, we seek the equivalent duty ratio as a function of the control variable $\left(D_{c}\right)$ and the ratio $V_{g} / I_{\text {out }}$. The average of $i_{\text {on }}$ is given by

$$
\bar{i}_{\mathrm{on}}=I_{\mathrm{on}}=\frac{1}{2} \frac{V_{\mathrm{off}}-\bar{v}_{d}}{L_{e}} D_{c} D_{1} T_{p} .
$$

The average voltage across every inductor has to be zero in steady state. Therefore,

$$
D_{c} V_{\text {off }}=D_{1} \bar{v}_{d}
$$

Using definition (6) and relation (11), (19) and (20) can be solved for the equivalent duty ratio:

$$
m=\frac{D_{c}^{2}}{\frac{I_{\mathrm{out}}}{I_{\alpha}}+D_{c}^{2}},
$$

where

$$
I_{\alpha} \equiv \frac{V_{g}}{2 L_{e} f_{s}} .
$$

An alternative expression for the dc conversion ratio,

$$
D_{c}=\sqrt{\frac{k \mathfrak{T} m(\mathfrak{T})}{1-m(\mathfrak{N})},}
$$

follows from the fact that $V_{g} / I_{\text {out }}=R / \mathfrak{T}$. Here, $R$ is the load resistance at dc,

$$
R \equiv V_{\text {out }} / I_{\text {out }}
$$

while $m(\mathfrak{N})$ is the solution of $M(m)=\mathfrak{T}$, as shown in Table I for three particular functions $M(D)$. Parameter $k$ lumps together all relevant circuit parameters,

$$
k \equiv \frac{2 L_{e} f_{s}}{R} .
$$

The dc characteristic given by (23) is in its inverse formthe control variable is found as a function of the conversion ratio. The explicit form, $\mathfrak{T}=M\left(D_{c}, k\right)$, can be obtained by inverting (23) for any given PWM converter with known $M(D)$. For the three conversion-ratio functions of basic PWM converters, the results summarized in Table II can be verified against the results found separately for specific converter topologies in [3].

Converters operate in DICM as long as $D_{1} \leq 1$ or, equivalently, as long as

$$
D_{c} \leq m \text {. }
$$

In the normalized load-to-output plane, this condition becomes

$$
\frac{I_{\text {out }}}{I_{\alpha}} \leq m(1-m)
$$

The operating regions for DICM and CCM and dc characteristics found from (21) are shown in Fig. 6. In CCM, the dc characteristics become horizontal lines, $m=D_{c}=$ $D$. At the boundary between DICM and CCM, parameter 
TABLE II

DC Conversion Ratio in DiCM Evaluated For the Most Common Conversion-Ratio Functions $M(D)$ in $\mathrm{CCM}$

\begin{tabular}{ccc}
\hline$M(D)$ & $D_{c}(\mathfrak{M}, k)$ & $M\left(D_{c}, k\right)$ \\
\hline$D$ & $\sqrt{\frac{k \mathbb{N}^{2}}{1-\mathfrak{M}}}$ & $\frac{2}{1+\sqrt{1+4 k / D_{c}^{2}}}$ \\
$\frac{1}{1-D}$ & $\sqrt{k \mathfrak{M}(\mathfrak{M}-1)}$ & $\frac{1+\sqrt{1+4 D_{c}^{2} / k}}{2}$
\end{tabular}

$\frac{D}{1-D} \quad \sqrt{k} \mathfrak{N} \quad \frac{D}{\sqrt{k}}$

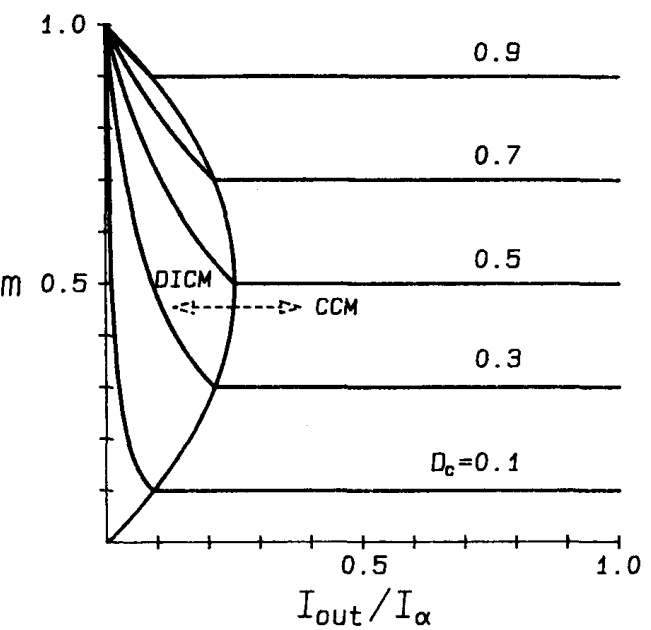

Fig. 6. DC characteristics of PWM converters in DICM and boundary between DICM and CCM in normalized load-to-output plane.

$k$ attains its critical value $k_{c}$ given by

$$
k_{c}=\frac{D_{c}\left(1-D_{c}\right)}{M\left(D_{c}\right)}
$$

and the converter operates in DICM if $k \leq k_{c}$. The entries in Table III agree with the results found separately for the buck, boost, and buck-boost converters in [3].

\section{Discontinuous Capacitor Voltage Mode}

The discontinuous capacitor voltage mode (DCVM) is dual to the discontinuous inductor current mode. Therefore, all results for DCVM follow directly from the results of the previous section and duality relations:

$$
\begin{aligned}
L & \leftrightarrow C, \\
i & \leftrightarrow v, \\
R & \leftrightarrow 1 / R, \\
m & \leftrightarrow 1-m, \\
D_{c} & \leftrightarrow 1-D_{c}, \\
\mathfrak{N} & \leftrightarrow 1 / \mathfrak{N} .
\end{aligned}
$$

TABLE III

Critical Value of the Parameter $k$ at THE BOUNDARY BETWEEN DICM AND CCM FOR THE THREE MOST COMMON Conversion-Ratio Functions $M(D)$ in CCM

\begin{tabular}{cc}
\hline$M(D)$ & $k_{c}$ \\
\hline$D$ & $1-D_{c}$ \\
$\frac{D}{1-D}$ & $\left(1-D_{c}\right)^{2}$ \\
$\frac{1}{1-D}$ & $D_{c}\left(1-D_{c}\right)^{2}$ \\
\hline
\end{tabular}

The equivalent duty ratio is

$$
m=1-\frac{\left(1-D_{c}\right)^{2}}{\frac{I_{\beta}}{I_{\text {out }}}+\left(1-D_{c}\right)^{2}},
$$

where

$$
I_{\beta} \equiv 2 V_{g} C_{e} f_{s}
$$

and

$$
\frac{1}{C_{e}} \equiv \sum_{\mathfrak{e}_{\text {off }}} \frac{1}{C_{i}} .
$$

Control variable $D_{c}$, as a function of the dc conversion ratio $\mathfrak{T}$, is given by

$$
D_{c}=1-\sqrt{\frac{k^{\prime}(1-m(\mathfrak{N}))}{\mathfrak{N} m(\mathfrak{M})}},
$$

where

$$
k^{\prime} \equiv 2 R C_{e} f_{s} .
$$

In the normalized load-to-output plane, the condition for operation in DCVM is

$$
\frac{I_{\text {out }}}{I_{\beta}} \geq \frac{1}{m(1-m)} .
$$

The critical value for parameter $k^{\prime}$ is given by

$$
k_{c}^{\prime}=D_{c}\left(1-D_{c}\right) M\left(D_{c}\right),
$$

and the converter operates in DCVM if $k^{\prime} \leq k_{c}^{\prime}$. The output-plane dc characteristics in CCM and DCVM, together with the boundary curve between the operating regions for CCM and DCVM, are shown in Fig. 7.

While any PWM converter enters DICM for a sufficiently low load current, a necessary and sufficient condition for the existence of DCVM is that $\mathfrak{C}_{\text {off }}$ is nonempty. Indeed, if $\mathcal{C}_{\text {off }}$ is empty, $V_{\text {off }}$ must be equal to $V_{g}$, and the PWM converter operates in CCM for an arbitrary large load current because $V_{g}$ is an ideal voltage source. However, if there exists a capacitor in the loop with the switches, the equivalent capacitance $C_{e}$ is finite and DCVM is entered for a sufficiently large load current $I_{\text {out }}$. 


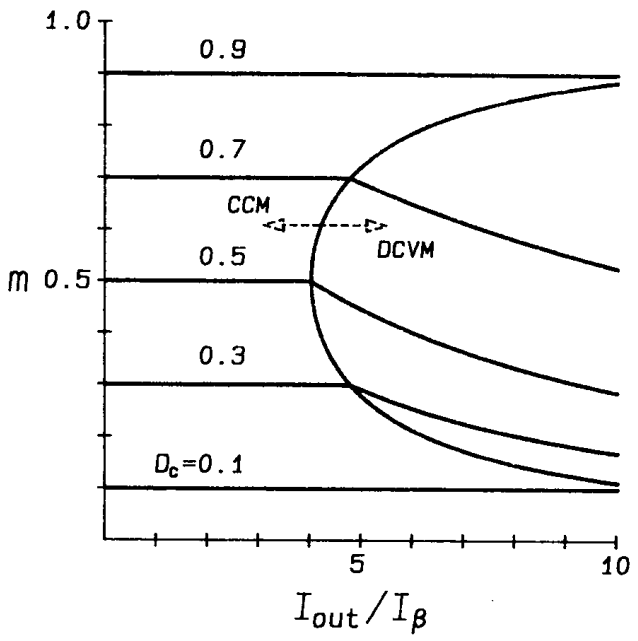

Fig. 7. DC characteristics and boundary between CCM and DCVM in normalized load-to-output plane.

\section{Discontinuous Quasi-Resonant Mode}

The dc analysis of DQRM is more involved because the operating mode includes a resonant state. Nevertheless, a closed form solution can be found for the equivalent duty ratio $m$ :

$$
m \frac{\theta_{p}}{\theta_{1}+\delta+\sqrt{\chi^{2}+\delta^{2}-1}+\frac{1}{2}\left(1-D_{c}\right)^{2} \theta_{p}^{2} \delta},
$$

where $\theta_{p}$ is the normalized switching period,

$$
\theta_{p} \equiv \frac{T_{p}}{2 \pi \sqrt{L_{e} C_{e}}},
$$

$\delta$ is the normalized output current,

$$
\delta \equiv \frac{I_{\text {out }}}{V_{g}} \sqrt{\frac{L_{e}}{C_{e}}},
$$

$\theta_{1}$ is the normalized length of the resonant (1-0)-state,

$$
\theta_{1}=\pi-\arctan \frac{\chi}{\delta}+\arctan \frac{1}{\sqrt{\chi^{2}+\delta^{2}}},
$$

and parameter $\chi$ is introduced for compactness,

$$
\chi \equiv\left(1-D_{c}\right) \delta \theta_{p}-1 \text {. }
$$

Conditions for operation in DQRM can be translated into the normalized load-to-output plane, with normalized switching frequency $f$,

$$
f \equiv 2 \pi \sqrt{L_{e} C_{e}} f_{s}
$$

as a varying parameter. The results are shown in Fig. 8. Only a limited range of equivalent duty ratios and a limited range of load currents are attainable while the converter operates in DQRM. This is not surprising because the converter in DQRM can be considered as a converter

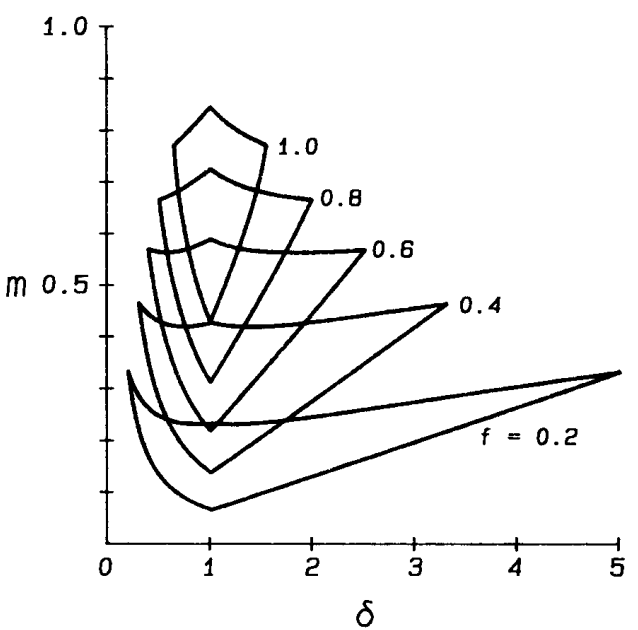

Fig. 8, Operating regions for PWM converters in DQRM in normalized load-to-output plane, with normalized frequency $f$ as varying parameter.

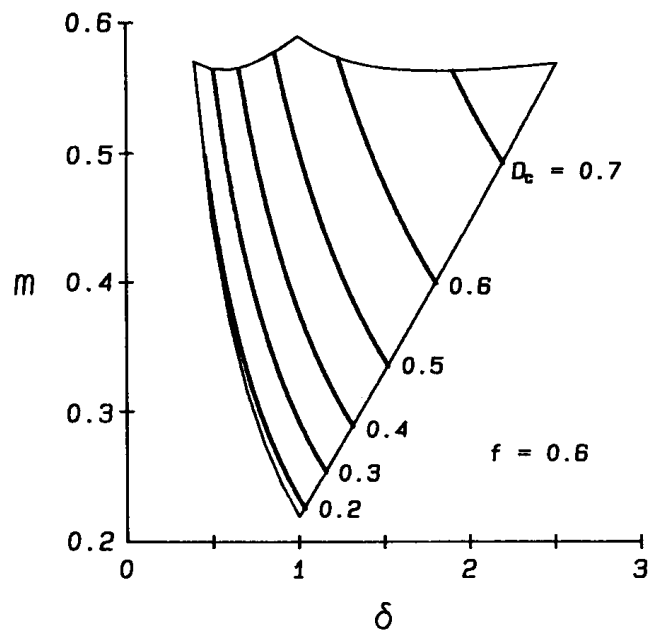

Fig. 9. Load-to-output dc characteristics of PWM converters in DQRM

that operates in DICM and DCVM simultaneously. Thus, qualitatively, the operating regions of Fig. 8 can be arrived at by overlapping the operating regions for DICM and DCVM. In the load direction, the operating region in DICM is bounded from above, while in DCVM, the operating region is bounded from below.

The normalized switching frequency $f$ is a free design parameter. It can be selected to adjust the available operating region according to a given set of design specifications. A choice that maximizes the load range for a given equivalent duty ratio is discussed in [9].

Load-to-output dc characteristics in DQRM are shown in Fig. 9 for $f=0.6$. The conversion ratio is strongly dependent on the load current, indicating that PWM converters in DQRM have a relatively large output resistance at dc. 


\section{AC Analysis}

In this section, we examine small-signal dynamic properties of PWM converters in discontinuous modes.

\section{A. A General Small-Signal Model}

Starting from the general dc model in Fig. 4, two steps are necessary in order to derive an averaged small-signal model capable of reproducing the behavior of PWM converters in discontinuous modes at low frequencies.

In the first step, we assume that an external variable$V_{g}, I_{\text {out }}$ or $D_{c}$-is modulated by a slowly-varying signal. The modulation frequency is sufficiently low so that effects of the sampling process can be ignored. Then, we postulate that the low-frequency spectrum of the averaged quantities faithfully represents the actual low-frequency spectrum of the circuit variables. This step is ubiquitous in derivations of averaged ac models, including the wellknown state-space-averaging model. As a result of this step, the equivalent duty ratio becomes a time-varying signal,

$$
m(t)=m\left(D_{c}(t), \frac{\bar{v}_{\mathrm{off}}(t)}{\bar{i}_{\mathrm{on}}(t)}\right)
$$

Relation (11) cannot be used to eliminate the internal variables $\bar{v}_{\text {off }}(t)$ and $\bar{i}_{\text {on }}(t)$ because it holds true only for the dc (steady-state) quantities.

The second step in the derivation is the standard smallsignal approximation-nonlinear relations are linearized around the dc operating point, so that the resulting model is linear and time-invariant. In the dc model of Fig. 4, all effects of the discontinuous mode are taken into account by substituting the duty ratio $D$ with the equivalent duty ratio $m$. The converter itself is treated as if it operated in the continuous conduction mode. Therefore, the second step in the derivation of the ac model results in the standard state-space averaged model of the converter in CCM (with the steady-state equivalent duty ratio $m$ instead of the steady-state duty ratio $D$ ), augmented by the feedback loops that represent the effects of the discontinuous mode of operation. The general ac model is shown in Fig. 10 . The feedback coefficients $\left(a_{c}, a_{i}\right.$ and $\left.a_{v}\right)$ are determined by the linearization of $(48)$ :

$$
\hat{m}=a_{c} \hat{d}_{c}+a_{i} \hat{\imath}_{\mathrm{on}}+a_{v} \hat{v}_{\mathrm{off}},
$$

where partial derivatives,

$$
\begin{aligned}
& a_{c} \equiv \frac{\partial m}{\partial d_{c}} \\
& a_{i} \equiv \frac{\partial m}{\partial i_{\mathrm{on}}} \\
& a_{v} \equiv \frac{\partial m}{\partial v_{\mathrm{off}}}
\end{aligned}
$$

are evaluated at the steady-state operating point

$$
m(t)=m, \quad \bar{v}_{\text {off }}(t)=V_{\text {off }}, \quad \bar{i}_{\text {on }}(t)=I_{\text {on }} .
$$

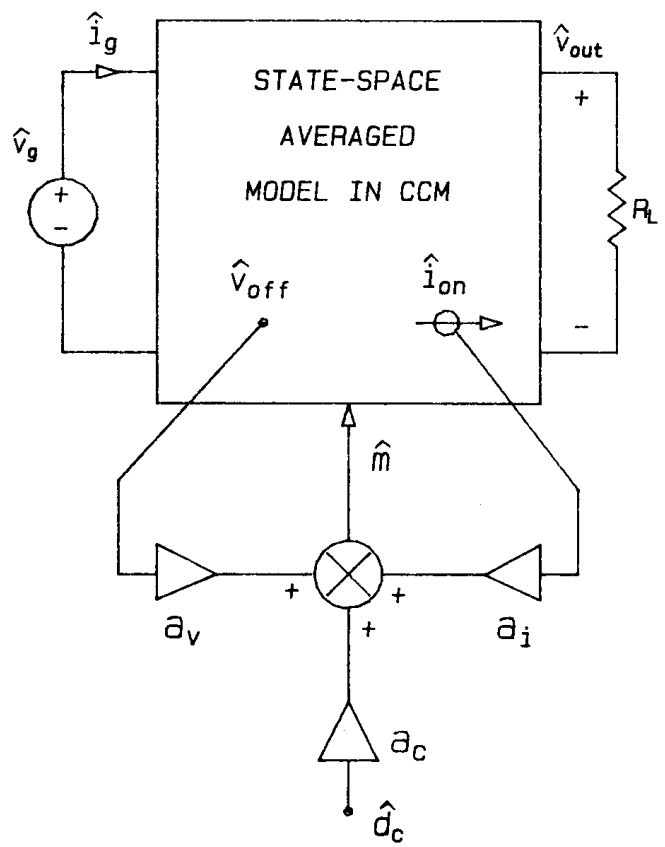

Fig. 10. General ac model of PWM converters in discontinuous modes

Some quite general conclusions follow immediately from the structure of the general ac model in Fig. 10:

1) The order of the model for a PWM converter operating in a discontinuous mode is the same as the order of the state-space averaged model for the same PWM converter operating in the continuous conduction mode.

2) Only poles of the control-to-output transfer function can be modified by the state feedback [10]. Therefore, in a discontinuous mode, zeros of the controlto-output transfer function $\left(\hat{v}_{\text {out }} / \hat{d}_{c}\right)$ are the same as the zeros of the control-to-output transfer function $\left(\hat{v}_{\text {out }} / \hat{d}\right)$ in the continuous conduction mode, except that the steady-state duty ratio $D$ is replaced by the steady-state equivalent duty ratio $m$. Therefore, zeros of the control-to-output transfer function exhibit identical dependence on the dc conversion ratio $\mathfrak{T}$, regardless of the mode of operation.

\section{B. State-Space Averaging in Discontinuous Modes}

General conclusions about the dynamic properties of PWM converters in the discontinuous modes are in contradiction with implications of the original state-space averaged model for DICM proposed in [3]. In this section, we demonstrate how the discrepancy can be completely removed if the dynamics of current $i_{\text {on }}$ is taken into account.

In [3], the standard averaged state-space model,

$$
\begin{aligned}
\dot{\boldsymbol{x}}= & \left(d_{c} \boldsymbol{A}_{1}+d_{2} \boldsymbol{A}_{2}+d_{3} \boldsymbol{A}_{3}\right) \boldsymbol{x} \\
& +\left(d_{c} \boldsymbol{b}_{1}+d_{2} \boldsymbol{b}_{2}+d_{3} \boldsymbol{b}_{2}\right) v_{g},
\end{aligned}
$$


is augmented by constraints

$$
\begin{aligned}
\frac{d i_{\mathrm{on}}}{d t} & =0, \\
i_{\mathrm{on}} & =i_{\mathrm{on}}\left(v_{\mathrm{off}}, d_{c}, L_{e}, T_{p}\right) .
\end{aligned}
$$

Matrices $\boldsymbol{A}_{i}$ and vectors $\boldsymbol{b}_{i}$ are the parameters of the statespace description

$$
\dot{\boldsymbol{x}}=\boldsymbol{A}_{i} \boldsymbol{x}+\boldsymbol{b}_{i} v_{g}
$$

for the three operating states of the converter in DICM. The original notation of reference [3] is slightly changed to be in agreement with the notation used in this paper. A small-signal ac model is derived after perturbation and linearization of (54) and (56). Note that the order of the model is reduced by one because the dynamics of $i_{\text {on }}$ is effectively ignored by imposing (55) as an additional constraint to the averaged state-space equations. If, however, this constraint is simply removed, and if the average of $i_{\text {on }}$ is computed over the complete switching cycle so that a relation

$$
\bar{i}_{\mathrm{on}}(t)=i_{\mathrm{on}}\left(\bar{v}_{g}, \bar{v}_{\mathrm{off}}(t), d_{\mathrm{c}}(t), d_{2}(t), L_{e}, T_{p}\right)
$$

is obtained, the state-space averaged model derived by perturbation and linearization of (54) and (58) becomes completely equivalent to the ac model of Section IV-A applied to the discontinuous inductor current mode.

\section{Discontinuous Inductor Current Mode}

From (21), the equivalent duty ratio is given by

$$
m=\frac{d_{c}^{2}}{k R\left(\bar{i}_{\text {on }} / \bar{v}_{\text {off }}\right)+d_{c}^{2}},
$$

so that the feedback coefficients are

$$
\begin{aligned}
& a_{c}=\frac{2 m(1-\mathrm{m})}{D_{c}}, \\
& a_{i}=-k \frac{Q(m) m^{2}}{D_{c}^{2}} \frac{R}{V_{g}}, \\
& a_{t^{\prime}}=k \frac{P(m) m^{2}}{D_{c}^{2}} \frac{1}{V_{g}} .
\end{aligned}
$$

The feedback parameters can be easily computed for any given PWM converter with known $M(D)=P(D) / Q(D)$, and for any given steady-state operating point. Moreover, the structure of the ac model in Fig. 10 allows us to derive some additional general results for the dynamics of PWM converters in DICM.

The control-to-output transfer function is defined by

$$
H_{c}(s) \equiv \frac{\hat{v}_{\text {out }}}{\hat{d}_{c}} K_{c} \frac{R(s)}{S(s)},
$$

where $K_{c}$ is the control-to-output dc gain. We have already shown that zeros of $R(s)$ are the same as zeros of the control-to-output transfer function in CCM in terms of the circuit parameters and the conversion ratio $\mathfrak{T}$. The

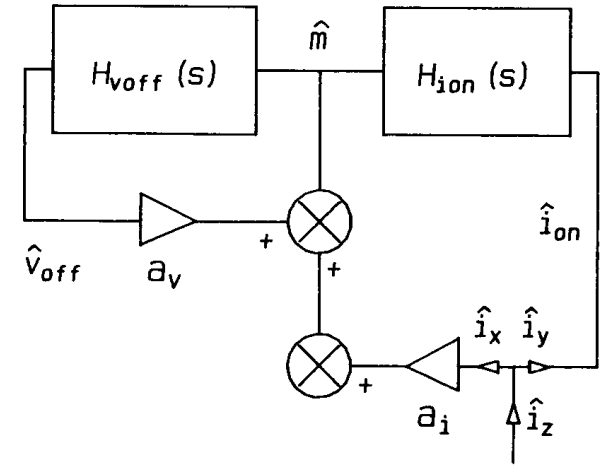

Fig. 11. Model for calculation of current loop gain $T_{c}=\hat{i}_{y} / \hat{i}_{x}$ by injection of current $\hat{i}_{-}$.

poles of the system's functions (zeros of $S(s)$ ) are affected by the feedback loops with gains $a_{i}$ and $a_{v}$.

High-Frequency Pole: The current feedback loop with gain $a_{i}$ introduces lossless damping into the ac response so that a pair of complex poles (zeros of $S(s)$ ) is split into two real poles. The effect is quite similar to the effect of the current-mode programming studied in [4]. Assuming that the real poles are well separated, we can proceed to find the location of the high-frequency pole. The ac model is redrawn in Fig. 11 in order to examine the loop gain of the inner current loop. Following the approach in [4], the voltage loop is nested inside the current loop, and a signal $\hat{i}_{z}$ is injected in order to calculate the current loop gain. The current loop gain $T_{c}(\mathrm{~s})$ is given by

$$
T_{c}(s)=\frac{\hat{i_{y}}}{\hat{i}_{x}}=-a_{i} H_{i \mathrm{on}}(s) \frac{1}{1-H_{i \text { off }}(s)},
$$

where $H_{\text {ion }}(s)$ is the transfer function from $\hat{m}$ to $\hat{i}_{\text {on }}$, and $H_{l \text { off }}(s)$ is the transfer function from $\hat{m}$ to $\hat{v}_{\text {off }}$. At high frequencies, $H_{v \text { off }}(s) \rightarrow 0$ and the dominant ac voltage across every inductor in $\mathscr{L}_{\text {on }}$ is equal to $V_{\text {off }} \hat{m}$, so that

$$
T_{c}(s) \rightarrow-a_{i} V_{\text {off }} \frac{1}{s L_{e}} \text { as } s \rightarrow \infty .
$$

By definition, $|T(j w)|$ is equal to 1 at the crossover frequency $w_{c}$ of the inner current loop. The crossover frequency can be found from (61) and (65):

$$
f_{c}=\frac{w_{c}}{2 \pi}=\frac{m^{2}}{D_{c}^{2}} \frac{f_{s}}{\pi} \text {. }
$$

As demonstrated in [4], the high-frequency pole of the system's transfer functions is located at the crossover frequency of the inner current loop. Since $m \geq D_{c}$ in DICM,

$$
f_{c} \geq \frac{f_{s}}{\pi} \text {. }
$$

Thus, the high-frequency pole approaches its lowest possible frequency, $f_{s} / \pi$, as the converter approaches the boundary of the continuous conduction mode where $m=$ 
$D_{c}$. As the converter moves deeper into the discontinuous mode, the high-frequency pole moves to even higher frequencies.

Control-to-Output DC Gain: In CCM, $V_{\text {out }}=M(D) V_{g}$, so that the de gain $K_{c}(\mathrm{CCM})$ of the control-to-output transfer function is

$$
K_{c}(\mathrm{CCM})=V_{g} M^{\prime}(D),
$$

where $M^{\prime}(D)$ is the derivative of $M(D)$ with respect to the duty ratio $D$. In DICM, dc gain $K_{c}$ is given by

$$
K_{c}=\frac{a_{c}}{1-a_{i} K_{i}-a_{l} K_{l}} M^{\prime}(m) V_{g},
$$

where $K_{t} \equiv d V_{\text {off }} / d m$ and $K_{i} \equiv d I_{\text {on }} / d m$ can be found from (9) and (10). The final result is

$$
K_{c}=2 V_{g} \frac{1}{1+\frac{D_{c}^{2}}{m^{2}} \frac{1}{k M^{\prime}(m)}} \frac{(1-m) D_{c}}{k m} .
$$

\section{Discontinuous Capacitor Voltage Mode}

All results for DCVM can be derived following the same steps as for DICM. The equivalent duty ratio is

$$
m=1-\frac{\left(1-d_{c}\right)^{2}}{\frac{k^{\prime}}{R}\left(\bar{v}_{\mathrm{off}} / \bar{i}_{\mathrm{on}}\right)+\left(1-d_{c}\right)^{2}},
$$

and the feedback coefficients are

$$
\begin{aligned}
& a_{c}=\frac{2 m(1-m)}{1-D_{c}} \\
& a_{i}=-k^{\prime} \frac{Q(m)(1-m)^{2}}{\left(1-D_{c}\right)^{2}} \frac{1}{I_{g}} \\
& a_{t^{\prime}}=k^{\prime} \frac{P(m)(1-m)^{2}}{\left(1-D_{c}\right)^{2}} \frac{1}{R I_{g}} .
\end{aligned}
$$

\section{E. Discontinuous Quasi-Resonant Mode}

Analytical expressions for the feedback coefficients in DQRM can be found from (42). Without going into the lengthy calculation (except numerically, the results would not provide any new information) one may conclude that the lossless damping that causes the separation of a pair of complex poles into a low-frequency and a high-frequency real pole is also present in DQRM because the dc characteristics are strongly load-dependent.

\section{F. An Example: The Boost Converter in DICM}

A model of the boost converter in DICM is shown in Fig. 12. The model illustrates one possible method for application of the general ac model of Fig. 10. The canonical circuit model of the boost converter in CCM [2] is augmented by the current and voltage feedback loops. Note also that the steady-state duty ratio $D$ is replaced by

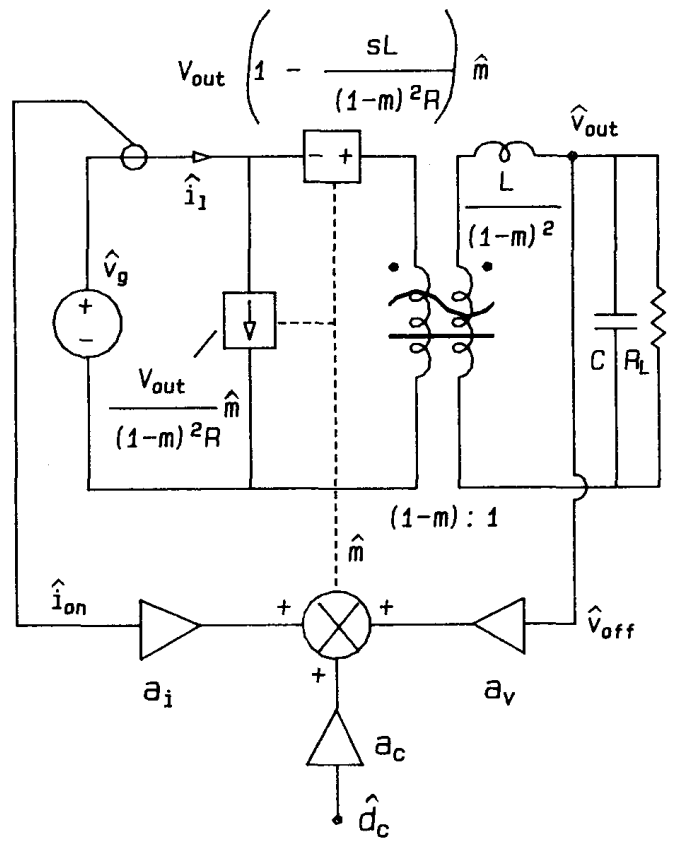

Fig. 12. Model of the boost converter in DICM.

the steady-state equivalent duty ratio $m$. The small-signal load resistance $R_{L}$,

$$
R_{L} \equiv \frac{\hat{v}_{\text {out }}}{\hat{i}_{\text {out }}}
$$

need not be equal to the de load resistance $R$. For simplicity, we shall assume that the load is linear so that $R_{L}$ $=R$.

The model of Fig. 12 can be used to derive analytic expressions for any system function of interest. Also, it can be used in any general-purpose circuit analysis program in order to simulate the small-signal dynamics of the converter in DICM.

As an example, we shall determine the control-to-output transfer function. Transfer functions $H_{i \text { on }}$ and $H_{i \text { off }}$ are easily determined from the model:

$$
\begin{aligned}
H_{\text {ion }}(s) & \equiv \frac{\hat{i}_{\mathrm{on}}}{\hat{m}} \\
& =K_{i} \frac{1+s / w_{z 1}}{1+\frac{1}{Q}\left(s / w_{o}\right)+\left(s / w_{o}\right)^{2}}, \\
H_{v \mathrm{off}}(s) & \equiv \frac{\hat{v}_{\mathrm{off}}}{\hat{m}} \\
& =K_{v^{\prime}} \frac{1-s / w_{z 2}}{1+\frac{1}{Q}\left(1 / w_{o}\right)+\left(s / w_{o}\right)^{2}},
\end{aligned}
$$


where

$$
\begin{aligned}
K_{i} & =\frac{2 V_{g}}{R(1-m)^{3}}, \\
w_{z 1} & =\frac{2}{R C}, \\
K_{l} & =\frac{V_{g}}{(1-m)^{2}}, \\
w_{z 2} & =\frac{R(1-m)^{2}}{L}, \\
w_{o} & =\frac{1-m}{\sqrt{L C}}, \\
Q & =R \sqrt{\frac{C}{L}} .
\end{aligned}
$$

Then, the control-to-output transfer function is given by

$$
H_{c}(s) \equiv \frac{\hat{v}_{\text {out }}}{\hat{d}_{c}}=\frac{a_{c} H_{m}(s)}{1-a_{i} H_{i \mathrm{on}}(s)-a_{v^{\prime}} H_{v^{\mathrm{off}}}(s)} .
$$

Note that

$$
H_{m}(s) \equiv \frac{\hat{v}_{\text {out }}}{\hat{m}}=H_{i \text { off }}(s),
$$

because $v_{\text {off }}=v_{\text {out }}$. From Eqs. (76), (77) and (84), we obtain

$$
H_{c}(s)=K_{c} \frac{1-s / w_{z}}{1+\alpha_{1} s+\alpha_{2} s^{2}},
$$

where

$$
\begin{aligned}
K_{c} & \equiv 2 V_{g} \frac{D_{c}}{k} \frac{1-m}{1+m}, \\
w_{z} & =\frac{L}{R(1-m)^{2}}, \\
\alpha_{1} & =\frac{m}{1+m} R C+\frac{1}{(1-m)^{2}} \frac{L}{R}, \\
\alpha_{2} & =\frac{D_{c}^{2}}{k m(1+m)} L C .
\end{aligned}
$$

The quadratic in the denominator can be factored out into two real poles and simple approximate solutions can be found if the poles are well separated, i.e., if $\alpha_{2} / \alpha_{1} \ll$ $\alpha_{1}$. This condition is satisfied if

$$
f_{s} \gg \frac{1}{D_{c}} \frac{1}{R C} \text {. }
$$

Note that condition (91) may not be satisfied for very low duty ratio $D_{c}$, even though the output $R C$ time constant is much larger than the switching period. However, in most cases, condition (91) will be satisfied and then,

$$
1+\alpha_{1} s+\alpha_{2} s_{2}=\left(1+\frac{s}{w_{p 1}}\right)\left(1+\frac{s}{w_{p 2}}\right)
$$

where

$$
\begin{aligned}
& w_{p 1} \approx \frac{1+m}{m} \frac{1}{R C}, \\
& w_{p 2} \approx k \frac{m^{2}}{D_{c}^{2}} \frac{R}{L}=2 \frac{m^{2}}{D_{c}^{2}} f_{s} .
\end{aligned}
$$

Frequency $f_{p 2}$

$$
f_{p 2} \equiv \frac{w_{p 2}}{2 \pi}=\frac{m^{2}}{D_{c}^{2}} \frac{f_{s}}{\pi}=\frac{\mathfrak{T}-1}{k \mathfrak{T}^{3}} \frac{f_{s}}{\pi},
$$

of the high-frequency pole is correctly predicted by the general result of (66).

Since

$$
f_{z} \equiv \frac{w_{z}}{2 \pi}=\frac{1}{2 \pi} \frac{L}{R(1-m)^{2}}=\frac{1}{\mathrm{kT}^{2}} \frac{f_{s}}{\pi},
$$

and because $\mathfrak{T}>1$ for the boost converter, the righthalf-plane zero is always at even higher frequency than $f_{p 2}$.

\section{G. Experimental Verification}

Control-to-output transfer function in DICM is measured for an experimental boost converter of Fig. 13. The results of the measurements (dashed lines) and the predictions (solid lines) are compared in Fig. 15(a)-(c) for the load $R=67.5 \Omega$ and three different values of the control duty ratio $D_{c}$. The values $D_{c}=0.1$ and $D_{c}=0.6$ are close to the boundary with CCM, while for $D_{c}=0.38$ the converter operates in deep DICM. A low-esr output capacitor is chosen to make sure that the contribution of the esr zero is negligible in the frequency range of interest. The resistor $(1 \mathrm{k} \Omega)$ in parallel with the inductor is included to damp the resonance between $L$ and the parasitic capacitances in the circuit. In all cases, it is evident that the phase response drops significantly below $-90^{\circ}$ predicted by the single-pole model. It is interesting that for $D_{c}=0.1$, close to the boundary with CCM, condition (91) is not satisfied and the two real poles are not well separated. In this case, the presence of the second pole is evident even in the magnitude response.

Another set of measurements was made for the Ćuk converter of Fig. 14 and the results are reported in Fig. 16(a)-(c). In the Ćuk converter,

$$
\mathcal{C}_{\text {off }}=\left\{C_{1}\right\} \text { and } \mathscr{L}_{\text {on }}=\left\{L_{1}, L_{2}\right\} \text {, }
$$

so that

$$
v_{\mathrm{off}}=v_{C 1} \text { and } i_{\mathrm{on}}=i_{L 1}+i_{L 2} \text {, }
$$

and

$$
L_{e}=L_{1} \| L_{2}
$$

The theoretical predictions (solid lines) were obtained using the SCAMP program developed by the Caltech's Power Electronics Group [11]. The program implements the state-space averaged method for the small-signal analysis of PWM converters in CCM, so that it supports the 


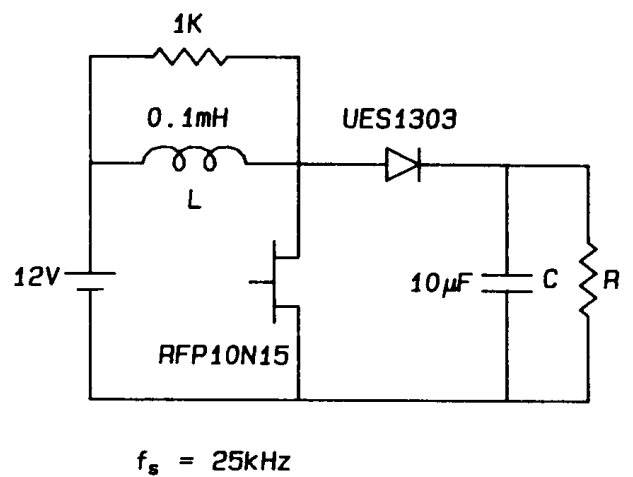

Fig. 13. Experimental boost converter.

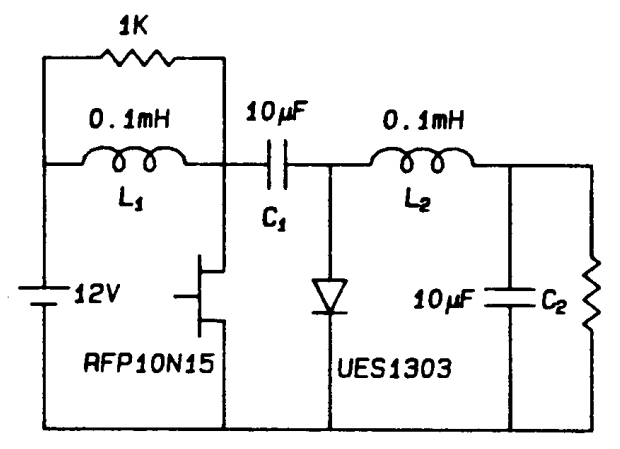

$f_{s}=25 \mathrm{KHz}$

Fig. 14. Experimental Ćuk converter.

ac model of Fig. 10 in a straightforward manner. This is yet another possibility for practical application of the general ac model proposed for PWM converters in discontinuous modes.

For the operating point in Fig. 16(a), the Cuk converter is in CCM and the measurement is included for easy comparison with the responses in DICM. As predicted by the state-space averaged model in CCM, the converter is a fourth-order system with two pairs of complex poles $\left(w_{p 12}\right.$, $\left.Q_{p 12}\right)$ and $\left(w_{p 34}, Q_{p 34}\right)$, and a pair of complex rhp zeros $\left(w_{z}, Q_{z}\right)$. The other two cases correspond to the operation in DICM-the operating point in Fig. 16(b) is close to the DICM/CCM boundary, while Fig. 16(c) corresponds to the operation far away from the boundary. In DICM, the predicted control-to-output transfer function has a pair of complex rhp zeros $\left(w_{z}, Q_{z}\right)$, a pair of complex poles $\left(w_{p 34}\right.$, $\left.Q_{p 34}\right)$ and a pair of real poles-a low-frequency pole $\left(w_{p 1}\right)$ and a high-frequency pole $\left(w_{p 2}\right)$. The corner frequencies and the $Q$-factors are indicated in Fig. 16 together with the steady-state operating conditions. Agreement between the measured and the predicted response is excellent.

\section{H. Limitations of the Averaged AC Model}

In the measurements of Figs. 15 and 16, the frequency range was intentionally limited. Because the sampling

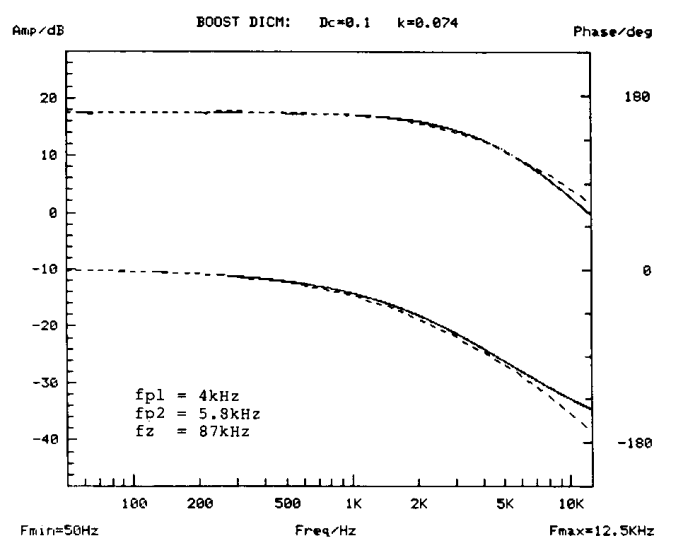

(a)

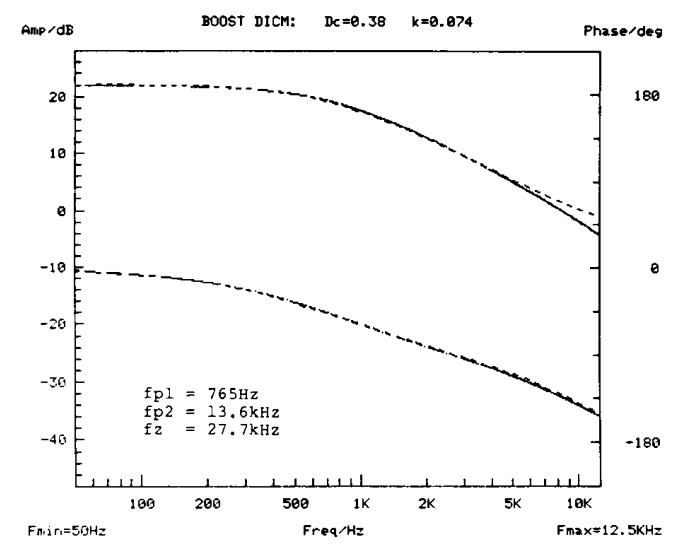

(b)

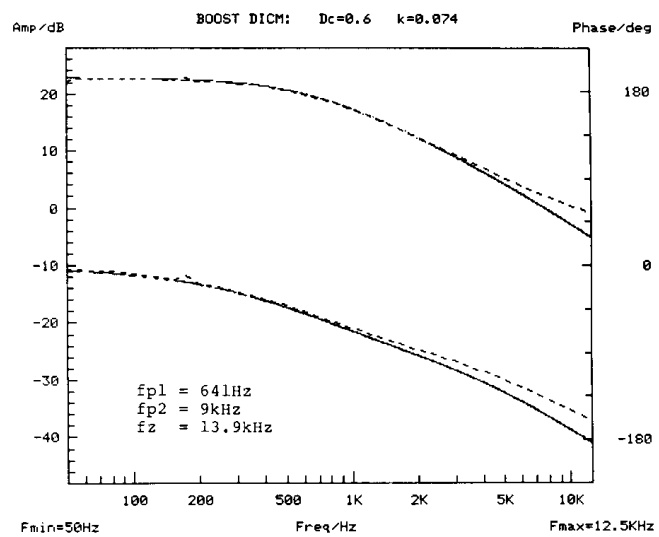

(c)

Fig. 15. Measured (dashed lines) and predicted (solid lines) frequency response of the boost converter of Fig. 13.

process is inherent to switched-mode converter systems, correct predictions of averaged models can be expected only if the response of the measured signals is bandlimited to at most one-half of the switching frequency. For 


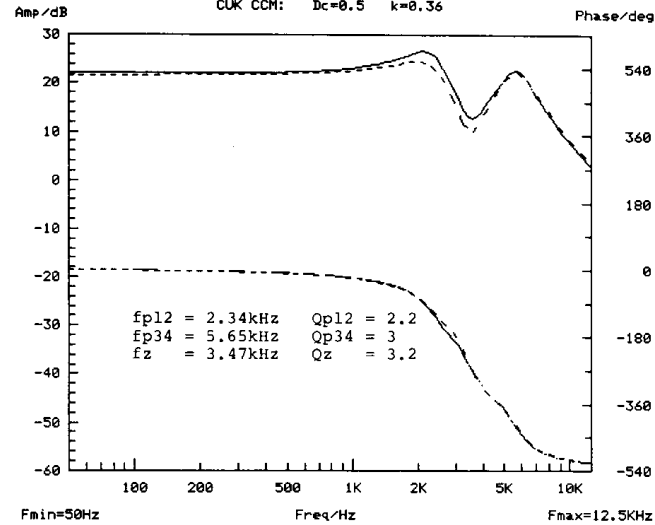

(a)

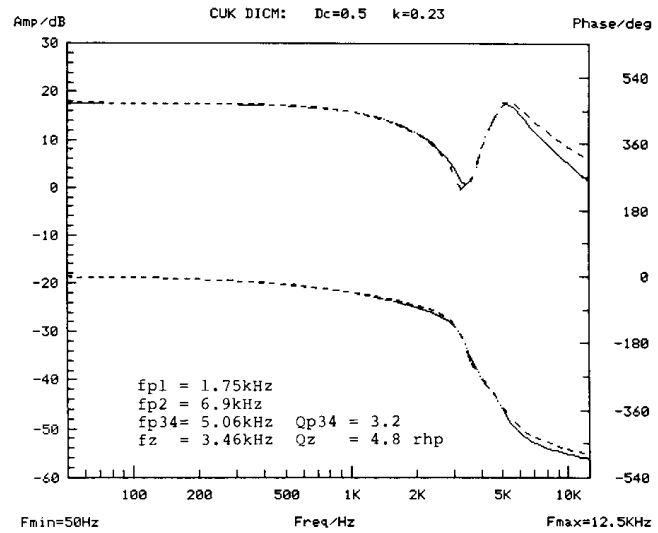

(b)

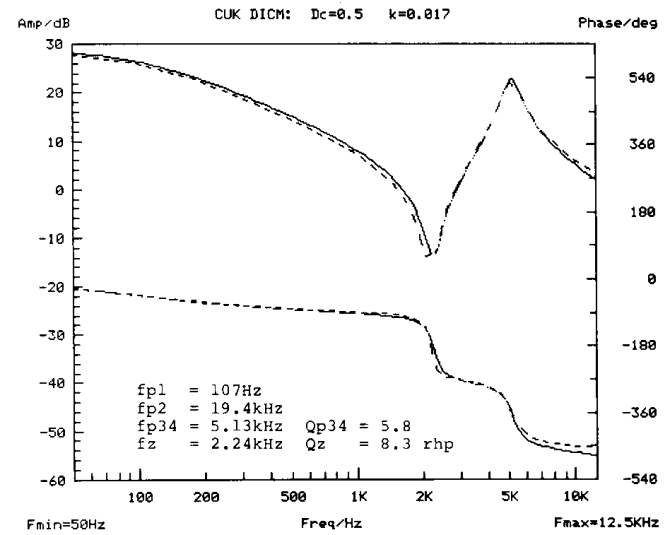

(c)

Fig. 16. Measured (dashed lines) and predicted (solid lines) frequency response of Ćuk converter of Fig. 14

the models of PWM converters in DICM, the agreement between the measured and the theoretical curves in Figs. 15 and 16 is quite satisfactory and in most cases excellent.
It is indicative, however, that in some cases the discrepancies observed in the frequency-response measurements of the experimental converters operating in DICM were larger than discrepancies commonly found in applications of the state-space averaged model in CCM. For the boost converter in particular, it was found that the actual phase lag of the control-to-output transfer function never exceeds $-180^{\circ}$, although the measurements were extended up to a frequency close to the switching frequency. On the other hand, the model predicts the total phase lag of $-270^{\circ}$ because there are two poles and a rhp zero in the transfer function.

There are two points worth noting with respect to validity of the averaged model for discontinuous modes. One of the assumptions in the derivation of the state-space averaged model is that the converter's corner frequencies are well below the switching frequency. With respect to the circuit waveforms, this assumption implies that ac ripples in capacitor voltages and inductor currents are relatively small. In the discontinuous modes, however, the ac ripple of at least one inductor current or capacitor voltage is relatively large. The second remark is concerned with the consistency between the premises and implications of the model. A general result derived from the averaged model (66) implies that the high-frequency pole of the converter in DICM is at the frequency higher than approximately one third of the switching frequency. Also, for the boost converter in DICM, it was shown that the predicted rhp zero is at even higher frequencies. Thus, the predicted corner frequencies are not well below the switching frequency, as originally assumed in the derivation of the model.

In conclusion, when averaged models are applied to cases in which the basic assumptions are not well satisfied and/or when the predicted corner frequencies are comparable to the switching frequency, the accuracy of the predicted response cannot be guaranteed, especially if the frequency range of interest is extended beyond one-half of the switching frequency. The relevant cases in which a complete accuracy can be guaranteed only by an exact sampled-data model (such as the model described in [12]) include not only PWM converters in discontinuous modes but also converters with the current-mode programming, resonant or quasi-resonant converters, etc. In the low-frequency range, which is of most interest for closing a feedback loop around the converter's power stage, the accuracy of the averaged model is quite satisfactory and its simplicity is the major advantage over the more accurate, sampled-data models.

\section{Conclusion}

A systematic study of possible operating modes of PWM converters revealed that in addition to the three known modes - the continuous conduction mode (CCM), the discontinuous inductor current mode (DICM), and the 
discontinuous capacitor voltage mode (DCVM)-there exists another mode-the discontinuous quasi-resonant mode (DQRM).

When a PWM converter operates in DQRM, four networks are repeatedly switched in every switching cycle. The transistor turn-ON and the diode turn-OFF are at zero current, while the transistor turn-OFF and the diode turnon are at zero voltage, so that switching losses are reduced, as in various quasi-resonant converters. Unlike quasi-resonant converters, however, the PWM converter in DQRM can be controlled just as PWM converters in CCM-by varying the duty ratio of the active switch at constant switching frequency. In addition, the active switch need not be two-quadrant.

A general dc and a general small-signal ac model are proposed for PWM converters in the three discontinuous modes. For a given mode, the model is unified for all basic PWM converters. Any particular topology is taken into account via its well-known dc conversion-ratio function $M(D)$ in CCM.

The $\mathrm{dc}$ model of the converter in the discontinuous mode (DCIM, DCVM or DQRM) duplicates its model in CCM except that the duty ratio $D$ is replaced by the equivalent duty ratio $m$. For each mode, the equivalent duty ratio is a function of the duty ratio of the active switch, the input voltage and the output current, but it does not depend on any particular converter topology. Analytic expressions for the equivalent duty ratio are found for all three discontinuous modes. For DICM and DCVM, the expressions confirm the results previously found for particular converter topologies, while the results for DQRM have not been reported before. It is shown that the operating region of the converter in DQRM is a rather restricted portion of the load-to-output plane. In all three cases, the equivalent duty ratio is a load-dependent function, which indicates that all PWM converters in the discontinuous modes exhibit a non-zero output resistance at dc.

In CCM, certain converter topologies exhibit the same dc conversion-ratio function $M(D)$. For example, $M(D)=$ $D /(1-D)$ for the buck-boost, Cuk and Sepic converters. The dc model, without any further calculation, implies that this property-the same dc conversion-ratio function-is carried over to all three discontinuous modes.

The averaged ac model incorporates the state-space averaged model of the power stage, just as if the power stage operated in CCM, except that the steady-state duty ratio $D$ is replaced by the steady-state equivalent duty ratio $m$. The effects of the discontinuous mode are taken into account via additional feedback loops with gains that depend on the steady-state operating point. The structure of the model implies two quite general conclusions, regardless of the operating mode and regardless of any specific topology. First, the order of the model in the discontinuous modes is the same as the order of the state-space averaged model in CCM and second, the zeros of the con- trol-to-output transfer function (including rhp zeros, as may be the case) in the discontinuous mode are the same as the zeros of the control-to-output transfer function in CCM. The zeros exhibit the same dependence on the circuit parameters and the dc conversion ratio, regardless of the mode of operation.

In the ac model, the load-dependence of the conversion ratio implies lossless damping in the dynamic response of the converter. The effect is similar to the effect of the current-mode programming on the power stage in $\mathrm{CCM}-\mathrm{a}$ pair of complex poles is split into a low-frequency and a high-frequency pole. The poles are well-separated if the converter operates away from the boundary with CCM, and if the output-filter time constant $(R C)$ is much larger than the switching period. In practice, the latter condition is always satisfied because the output voltage must have a low ac ripple. In DICM, if the poles are well-separated, the high-frequency pole is higher than approximately onethird of the switching frequency $\left(f_{s} / \pi\right)$. This result is also quite general-it is derived without reference to any particular PWM topology. The effect of the high-frequency pole is quite noticeable in the measured responses and the measurements confirm that the full-order averaged model provides improved predictions when compared to the earlier, reduced-order state-space-averaged model for DICM.

Since the suggested ac model includes the well-known state-space averaged model for CCM, there is a variety of possible application methods. Equivalent-circuit modeling can start from already available canonical models of the power stage in CCM and then, the circuit model can be used to derive analytic results or in a general-purpose circuit-analysis program; known analytic results for transfer functions of PWM converters in CCM can be used in a block-diagram approach; finally, the model can be readily utilized in conjunction with the SCAMP software [11], or any other computer program originally designed for the small-signal analysis of PWM converters in the continuous conduction mode.

\section{ACKNOWLEDGMENT}

The assistance is gratefully acknowledged of Ljubiša Stevanović, a graduate student at California Institute of Technology and a member of the Power Electronics Group, for the help in constructing the experimental circuits and producing the experimental results in Section IV.

\section{REFERENCES}

[1] S. Cuk, "General topological properties of switching structures," in IEEE PESC, 1979 Rec, pp, 109-130.

[2] R. D. Middlebrook and S. Cuk, "A general unified approach to modelling switching converter power stages,'” in IEEE PESC, 1976 Rec., pp. 18-34.

[3] S. Cuk and R. D. Middlebrook, "A general unified approach to mod- 
elling switching dc-to-dc converters in discontinuous conduction mode, " in IEEE PESC, 1977 Rec., pp. 36-57.

[4] R. D. Middlebrook, "Topics in multiple-loop regulators and currentmode programming," in IEEE PESC, 1985 Rec., pp. 716-732.

[5] K-H. Liu and F. C. Lee, "Resonant switches-A unified approach to improve performances of switching converters," in IEEE International Telecommunications Energy Conf., 1984 Proc., pp. 344-351.

[6] K-H. Liu and F. C. Lee, "Zero-voltage switching technique in dc/ dc converters," in IEEE PESC, 1986 Rec., pp. 58-70.

[7] D. Maksimović and S. Cuk, "A general approach to synthesis and analysis of quasi-resonant converters," in IEEE PESC, $1989 \mathrm{Rec}$. pp. 713-727.

[8] S. Freeland and R. D. Middlebrook, "A unified analysis of convert ers with resonant switches," in IEEE PESC 1987 Rec., pp. 20-30.

[9] D. Maksimović, "Synthesis of PWM and quasi-resonant dc-to-dc power converters," Ph.D. dissertation, California Institute of Technology, Pasadena, 1989

[10] C.-T. Chen, Linear System Theory and Design. New York: Holt, Rinehart and Winston, 1985, pp. 334-340.

[11] S. Ćuk, B. Lau, R. Gupta, and D. Borković, "Computer aided design of switching converter frequency response," in Power CAD '87 Conf. Rec.

[12] B. Lau and R. D. Middlebrook, "Small-signal frequency response theory for piecewise-constant two-switched-network dc-to-dc converter systems," IEEE PESC, 1986 Rec., pp. 186-200.

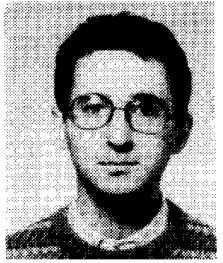

Dragan Maksimović (M'89) received the B.S. and M.S. degrees from the University of Belgrade, Belgrade, Yugoslavia, and the Ph.D. degree from California Institute of Technology, Pasadena, in 1984,1986 , and 1989 , respectively, all in electrical engineering.

$\mathrm{He}$ is currently Assistant Professor of Electrical Engineering, Faculty of Electrical Engineering, University of Belgrade. His research interests include modeling, control and design of power and signal processing systems.

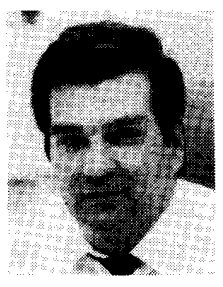

Slobodan Cuk (M`74) received the B.S. degree from the University of Belgrade, Belgrade, $\mathrm{Yu}$ goslavia, the M.S. degree from the University of Santa Clara, Santa Clara, CA, and the Ph.D. degree from California Institute of Technology, Pasadena, all in electrical engineering, in 1970, 1974. and 1977 , respectively.

$\mathrm{He}$ is Associate Professor of Electrical Engineering at the California Institute of Technology, Pasadena, where he conducts research in power electronics. 